\title{
Séminaires / Seminari 2011
}

\section{Ouverture et reprise d'un cabinet médical}

\section{Contenu}

Business plan (préparation du plan de financement et crédit d'exploitation, financement par la banque), Aménagement (implantation, projet et concept d'aménagement, choix du mobilier, budget), Estimation d'un cabinet (inventaire et goodwill), Administration d'un cabinet médical (dans le cabinet, par la banque), Assurances (toutes les assurances à l'intérieur et autour du cabinet), Passage du statut de salarié à celui d'indépendant et fiscalité.

\section{Sponsors}

Les coûts sont pris en charge par divers sponsors (voir www.fmhservices.ch).

\section{Dates}

\begin{tabular}{lll} 
K20 & $\begin{array}{l}\text { Jeudi, } 10 \text { mars } 2011 \\
13.30-18.00 \mathrm{~h}\end{array}$ & $\begin{array}{l}\text { World Trade } \\
\text { Center Lausanne }\end{array}$ \\
K21 & $\begin{array}{l}\text { Jeudi, } 26 \text { mai } 2011 \\
13.30-18.00 \mathrm{~h}\end{array}$ & $\begin{array}{l}\text { Ramada Park } \\
\text { Hôtel Genève }\end{array}$ \\
\hline
\end{tabular}

\section{Remise d'un cabinet médical}

\section{Contenu}

Aspects juridiques (autour du contrat de remise/ reprise), Estimation d'inventaire et goodwill d'un cabinet, Assurances (prévoyance, assurances à l'intérieur et autour du cabinet), Conséquences fiscales d'une remise.

\section{Sponsors}

Les coûts sont pris en charge par divers sponsors (voir www.fmhservices.ch).

\section{Dates}

$\begin{array}{lll}\text { K24 } & \begin{array}{l}\text { Jeudi, } 5 \text { mai } 2011 \\ 17.00-21.30 \mathrm{~h}\end{array} & \begin{array}{l}\text { World Trade } \\ \text { Center Lausanne }\end{array} \\ \text { K25 } & \begin{array}{l}\text { Jeudi, } 17 \text { novembre 2011 } \\ 17.00-21.30 \mathrm{~h}\end{array} & \begin{array}{l}\text { Ramada Park } \\ \text { Hôtel Genève }\end{array}\end{array}$

\section{Apertura e rilevamento di uno studio medico}

\section{Contenuto}

Business plan (preparazione del piano di finanziamento e del credito d'esercizio, prestito bancario), Pianificazione (insediamento, progetto e pianificazione, scelta del mobilio, budget), Valutazione di uno studio medico (inventario e goodwill), Ammini- strazione di uno studio medico (interna allo studio, rapporti con la banca), Assicurazioni (tutte le assicurazioni necessarie interne ed esterne allo studio), Passaggio dallo stato di dipendente a quello di indipendente, fiscalità.

\section{Documentazione}

Basato su una check-list per l'apertura di uno studio medico, il raccoglitore, consegnato ad ogni partecipante, contiene tutte le informazioni utili durante e dopo il seminario.

\section{Sponsor}

Diversi sponsor si fanno carico delle spese (si rimanda al sito www.fmhservices.ch).

\section{Date}

$\begin{array}{lll}\text { K50 } & \begin{array}{l}\text { Martedì, } 1 \text { marzo } 2011 \\ \text { dalle } 17.00 \text { alle } 21.00\end{array} & \begin{array}{l}\text { FMH Fiduciaria } \\ \text { Services Chiasso }\end{array} \\ \text { K51 } & \begin{array}{l}\text { Giovedì, } 12 \text { maggio } 2011 \\ \text { dalle } 14.00 \text { alle } 18.00\end{array} & \begin{array}{l}\text { FMH Fiduciaria } \\ \text { Services Chiasso }\end{array}\end{array}$

\section{Inscription et information / \\ Iscrizioni e informazioni}

www.fmhservices.ch oder FMH Consulting Services, Cornelia Steinmann, Burghöhe 1, 6208 Oberkirch, Tel. 04192500 77, Fax 0419210586.

\section{Remarques / Osservazioni}

Les adresses des participants aux séminaires dont les coûts sont couverts en partie ou totalement par des sponsors sont communiquées aux sponsors concernés.

Gli indirizzi dei partecipanti ai seminari, i cui costi sono coperti in parte o completamente da degli sponsor, vengono comunicati agli sponsor interessati.

\section{Conditions d'annulation / \\ Condizioni d'annullamento}

Un montant est perçu pour une absence ou une annulation. Il est de:

Un importo verrà rimborsato in caso di assenza o annullamento. Esso sarà di:

- 50 CHF par personne dans les 15 jours avant le début du séminaire / per persona entro i 15 giorni prima dell'inizio del seminario;

- 100 CHF par personne dans les 7 jours avant le début du séminaire / per persona entro i 7 giorni prima dell'inizio del seminario. 